\title{
A Review on IEEE 802.11p for Intelligent Transportation Systems
}

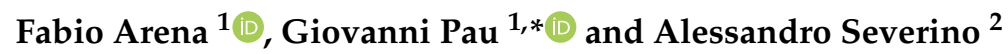 \\ 1 Faculty of Engineering and Architecture, Kore University of Enna, 94100 Enna, Italy; fabio.arena@unikore.it \\ 2 Department of Civil Engineering and Architecture, University of Catania, 95123 Catania, Italy; \\ alessandro.severino@unict.it \\ * Correspondence: giovanni.pau@unikore.it
}

Received: 15 February 2020; Accepted: 24 April 2020; Published: 26 April 2020

\begin{abstract}
Road safety is an active area of research for the automotive industry, and certainly one of ongoing interest to governments around the world. The intelligent transportation system (ITS) is one of several viable solutions with which to improve road safety, where the communication medium (e.g., among vehicles and between vehicles and the other components in an ITS environment, such as roadside infrastructure) is typically wireless. A typical communication standard adopted by car manufacturers is IEEE $802.11 \mathrm{p}$ for communications. Thus, this paper presents an overview of IEEE 802.11p, with a particular focus on its adoption in an ITS setting. Specifically, we analyze both MAC and PHY layers in a dedicated short-range communication (DSRC) environment.
\end{abstract}

Keywords: IEEE 802.11p; DSRC; intelligent transportation system; road safety

\section{Introduction}

Vehicle manufacturers and government agencies have been exploring and researching ways to improve road safety, more effectively manage traffic flows, etc. [1-3]. With the advancement in wireless communication systems [4-7], the vision of vehicles communicating with each other and with other devices (e.g., mobile devices and roadside units) situated at various locations (e.g., roads and buildings) is becoming a reality [8-10]. For instance, an Internet-connected device (e.g., a mobile device/application) could notify drivers of the proximity of a bicyclist in the street or a pedestrian on a crosswalk [11-15], which could potentially reduce the number of pedestrian-related accidents. Such intelligent or smart safety systems have been referred to in the literature as vehicle-to-vehicle (V2V; or car-to-car (C2C)), vehicle-to-infrastructure (V2I), vehicle-to-everything (V2X; or car-to-everything $(\mathrm{C} 2 \mathrm{X})$ ), vehicular ad hoc networks (VANETs), and so on [16-18].

All these terminologies can be assorted into a particular extensive research area denominated intelligent transportation systems (ITS) [19-25]. The main aim is to suggest innovative and smart solutions based on information and communication technologies (ICT) [26-30]. For instance, autonomous driving, which is cause for considerable excitement now, can also be facilitated by ITS, whose applications range from infotainment to automated and connected vehicles. These applications can be safety-related or not. It is useful to note that reliability and low-latency in communications are the most crucial necessities in numerous ITS safety applications.

One key role of intelligent or smart safety systems is traffic control, based on (real-time) analysis of data from different sources (e.g., vehicles or other devices, and different content or service providers) [31-35] relevant to the road and other conditions (e.g., weather) [36]. A more efficient and effective traffic control system will also result in reduced carbon emissions, for instance. Similarly to most technologies and systems, ITS systems differ in terms of features and functionalities, based on user and societal needs, regional and regulatory factors, and so on. Table 1 presents some works in the 
literature focused on revising several features of the IEEE 802.11p protocol. The analysis of these works has highlighted that, typically, ITS systems use IEEE 802.11p [37] for the MAC and PHY layers [38-43]. At the upper levels, services are provided by protocols designed by different working groups, such as IEEE 1609.2, IEEE1609.3, and IEEE 1609.4. These protocols relate to vehicle communications, which have also been referred to as wireless access in vehicular environments (WAVE).

Table 1. Existing IEEE 802.11p analyses/reviews/surveys: a comparative summary.

\begin{tabular}{cccccc}
\hline Contribution & $\begin{array}{c}\text { DSRC } \\
\text { Analysis }\end{array}$ & $\begin{array}{c}\text { PHY } \\
\text { Analysis }\end{array}$ & $\begin{array}{c}\text { MAC } \\
\text { Analysis }\end{array}$ & $\begin{array}{c}\text { ITS } \\
\text { Analysis }\end{array}$ & $\begin{array}{c}\text { Scope of } \\
\text { Literature Review }\end{array}$ \\
\hline Our review & Yes & Yes & Yes & Yes & $2006-2019$ \\
{$[39]$} & No & Yes & No & No & $2000-2018$ \\
{$[40]$} & No & Yes & No & Yes & $2004-2016$ \\
{$[41]$} & Yes & Yes & Partial & Partial & $2000-2016$ \\
{$[42]$} & Yes & Yes & Yes & Partial & $2000-2010$ \\
{$[43]$} & Yes & Yes & Yes & Partial & $2010-2017$ \\
\hline
\end{tabular}

In this paper, we provide an overview of the IEEE 802.11p, with a particular focus on ITS. The development of the IEEE $802.11 \mathrm{p}$ standard has also been partly attributed to advances in dedicated short-range communication (DSRC), which allows one to provide communication services between vehicles and road infrastructures; e.g., V2V [44,45] and V2I [46,47]. In other words, the IEEE 802.11p is a set of protocol architectures, whose primary purpose is to guarantee road safety and to manage traffic better.

The remainder of this review paper is organized as follows. Section 2 presents relevant preliminary materials concerning DSRC. MAC and PHY features of IEEE 802.11p are respectively analyzed in Sections 3 and 4. Section 5 presents a brief comparison between some features of IEEE 802.11p and LTE-V2X. Section 6 presents some features of the protocol which should be the evolution of IEEE $802.11 \mathrm{p}$, and finally, Section 7 concludes this paper.

\section{Preliminary Notes on Dsrc}

DSRC is a standard proposed by the Federal Communication Commission (FCC), which, in 1999, reserved $75 \mathrm{MHz}$ of bandwidth in the $5.9 \mathrm{GHz}$ frequency $(5.850-5.925 \mathrm{GHz})$ to support both V2V and V2I communications. A DSRC system consists of an on board unit (OBU) and a road side unit (RSU). The term short range indicates a coverage of hundreds of meters-less than, for instance, that of mobile phones [48,49].

The IEEE 802.11p standard has a set of specifications useful for making communication possible in the vehicular environment; that is, in an environment where there are rapid changes. The operating frequency of WAVE is fixed in the DSRC, the $5.85-5.925 \mathrm{GHz}$ band. Within this range, there is a channel reserved for system control and safety-related messages, and it takes the name of CCH (control channel); and up to six channels are used for the exchange of non-safety data, signaling channels (SCHs). WAVE conceives an alternate access scheme: the channel time is divided into synchronization intervals with a fixed length of $100 \mathrm{~ms}$, consisting of intervals of equal length alternating between the $\mathrm{CCH}$ and the $\mathrm{SCH}$. During the $\mathrm{CCH}$ interval, all vehicular devices must tune in to the $\mathrm{CCH}$ frequency for safety-related and system control data exchange; during the $\mathrm{SCH}$ interval, vehicles (optionally) switch to one of the SCH frequencies. At the beginning of each interval, a guard time of $4 \mathrm{~ms}$ is set to take into account the radio switching delay and timing inaccuracies in the devices. Coordination between channels uses Coordinated Universal Time (UTC) for a global time reference provided by a global satellite navigation system.

As shown in Figure 1, the DSRC spectrum is arranged in seven $10 \mathrm{MHz}$ channels. The guard band is $5 \mathrm{MHz}$; i.e., a part of the DSRC spectrum not employed to prevent interference. Channel 178 $(5.885-5.895 \mathrm{GHz})$ is reserved solely for safety communications and is termed the control channel $(\mathrm{CCH})$; it carries the most critical alarms and beacons. It is the unique channel shared between all the 
WAVE devices, and consequently, constitutes a joining point between the nodes. The two channels at the edge of the spectrum are reserved for prospective applications and particular employment, such as advanced accident prevention and public safety uses. The remaining channels are service channels $(\mathrm{SCH})$ for the residual applications and regular communications. Pairs of these adjacent channels can also be blended in a $20 \mathrm{MHz}$ channel. Nevertheless, the necessity to have $10 \mathrm{MHz}$ channels is associated with the use of parallel applications, and as a consequence, the partial reduction of channel congestion. Besides, physical tests prove that $10 \mathrm{MHz}$ channels are more suitable both for delays and the Doppler effect ascertained in vehicular environments. The DSRC band is subject to limitations and governed by several rules, such as the application of a specific one standard, unlike the Industrial Scientific and Medical (ISM) bands (900 MHz, 2.4 GHz, and $5 \mathrm{GHz}$ ) which are not licensed.

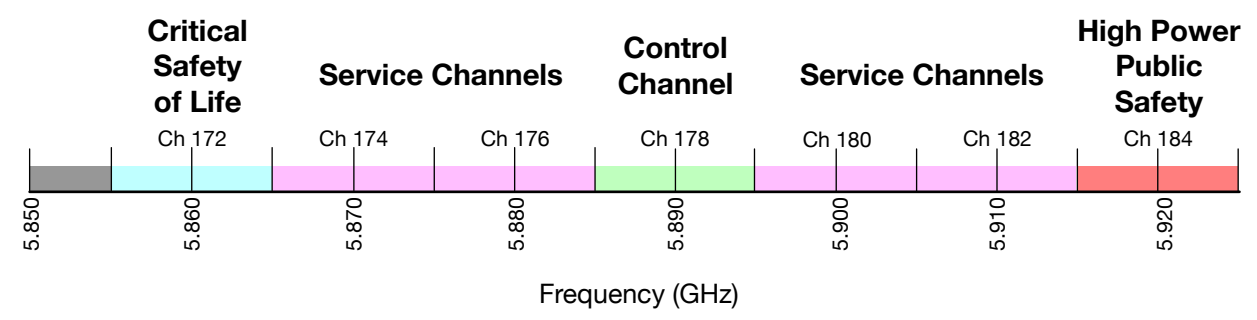

Figure 1. Dedicated short-range communication (DSRC) spectrum.

In the DSRC architecture (Figure 2), each layer practices different protocols. For the PHY and MAC layers, the IEEE 802.11p is adopted, while at the upper layers the IEEE 1609.2, 1609.3, and 1609.4 protocols are implemented, each of which deals explicitly with some services. In particular, they are respectively: security services (1609.2), network services (1609.3), and channel switching (1609.4). In the network layer, the WAVE Short Message Protocol (WSMP) is employed, but, as shown in Figure 2, depending on the application, it is also permissible to utilize other protocols, such as IPv6, $\mathrm{TCP}$, and UDP.

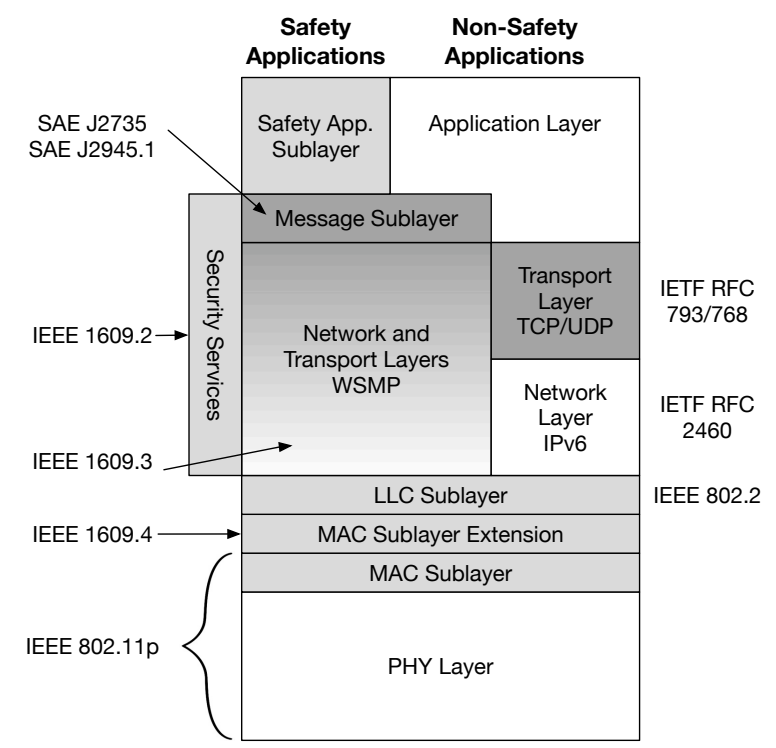

Figure 2. DSRC architecture.

The WAVE architecture includes IEEE P1609.1 (application layer), IEEE P1609.2 (security layer), IEEE P1609.3 (network layer), IEEE P1609.4 (upper MAC Layer), and IEEE 802.11p (lower MAC and physical layers). The SAE J2735 standard defines message sets, data-frames, and data-elements used by applications to exchange data over the DSRC/WAVE, and other communication protocols. Moreover, the SAE J2735 includes the following message categories: general, safety, geolocation, traveler information, and electronic payment. 
The IEEE $802.11 p$ outlines an unfolding of the IEEE 802.11 protocol. Changes have been made regarding the MAC and PHY layers to support different classes of vehicular communications. Since the IEEE 802.11p works on $5.9 \mathrm{GHz}$, the modifications have been principally applied to the most related standard, i.e., the IEEE 802.11a, which works at frequencies around $5 \mathrm{GHz}$. It is beneficial to perceive that the IEEE $802.11 \mathrm{p}$ has two precise purposes:

- Describe the functions and services required by the WAVE systems;

- Define the interfaces that allow communication with higher layers.

The DSRC standard is essentially an ad-hoc adjustment of the IEEE 802.11 aimed at reducing, as much as possible, the size of the packet to be sent via the radio link. The architecture of the IEEE $802.11 \mathrm{p}$ is an adjustment to the IEEE 802.11, although it can be considered as a new architecture. It is entirely based on the previous IEEE 802.11a protocols and the main features of the IEEE 802.11q. The main changes were made at the MAC layer, as it was necessary to intervene to make communication between vehicles faster, and at the same time, efficient, also because V2V or V2I communications can also take place at high speeds, and therefore, the communication time must be able to be very short.

\section{Mac Layer}

It is useful to appraise the differentiation between basic service set (BSS), service set identifier (SSID), and basic service set identifier (BSSID) to estimate the adjustments to the MAC layer introduced in IEEE 802.11p. A BSS is the fundamental part of the IEEE 802.11 standard and consists of a set of stations that can communicate with each other. An SSID represents the name with which the network distinguishes itself and is communicated, for instance, through a beacon frame by an access point (AP) in the case of BSS infrastructure. On the contrary, the BSSID recognizes the MAC address, composed of 6 bytes, of the access point.

A new feature introduced in IEEE 802.11p is represented by the WAVE mode [50-53]. Vehicles can interact with each other with a diminished overhead because they practice the same BSSID wildcard and the same channel. In the WAVE mode, the station can transmit and receive data frames with a BSSID wildcard regardless of its wave basic service set (WBSS). The latter consists of a set of stations (vehicles) working in WAVE mode that employs a common BSSID. The endowment of a WBSS befalls when a station in WAVE mode transfers a WAVE beacon comprising information on the type of service and parameters required for auto-configuration. Just one exchange of the WAVE beacon must be sufficient to warrant more numerous vehicles to join the WBSS. Anyhow, it is beneficial to heed that a station cannot belong to more than one WBSS. The WAVE beacon is obtained by the stations and transferred to others to raise the number of devices belonging to the WBSS. Hence, the latter is formed by a vehicle that requests it through the WAVE beacon and remains active even if the first vehicle is no longer in existence. On the contrary, the WBSS desists when there are no more vehicles that make it up. The stations use the BSSID wildcard for secure communications. Moreover, the BSSID wildcard is received by all stations, even without the obligation to belong to a specific WBSS.

The MAC layer is commensurate to the EDCA (enhanced distributed channel access) of IEEE 802.11e [54]. The ECDA keeps not only the feedback mechanisms represent by the ACK but also the request to send/clear to send (RTS/CTS) process. Moreover, it adds the possibility of exploiting a transmission opportunity (TXOP), and as a consequence, the messages are listed in different access categories (ACs), where $\mathrm{AC} 0$ has the lowest priority and $\mathrm{AC} 3$ the highest.

At the MAC layer, carrier sensing multiple access with collision avoidance (CSMA/CA) is adopted to make sure that a node that needs to transmit detects the means to verify if it is free. A random backoff mechanism is also used to reduce the likelihood of collisions. In vehicular communications, the recognition is not envisaged, and therefore, the exponential backoff mechanism, introduced in the other versions, is not applied. However, the CSMA/CA introduces significant overhead, especially in the presence of short packets and high-level modulations. Furthermore, the communications are 
known to be severely affected by collisions when channel occupancy increases by $50-60 \%$, a condition during which mechanisms are needed to avoid overloading [55]. To complete the analysis of the MAC layer, it is useful to analyze two phenomena that often lead to a decrease in performance in vehicular communications: the hidden terminal and the capture effect. The problem of hidden terminals is often indicated as the main factor of performance degradation within ad-hoc vehicle networks [56]. As demonstrated in ref. [57], it is possible to verify that by comparing two different methods of control of average access, i.e., CSMA and self-organizing time division multiple access (STDMA), the results reveal that the presence of hidden terminals does not significantly affect the performance of the two MAC protocols. However, the STDMA bestows a higher probability of receiving packets for all settings due to the synchronized packet transmissions. The phenomenon of frame capture, sometimes also referred to as capture echo, is the event that IEEE 802.11 chipsets show when dealing with frame collisions. Depending on the differences in the precise arrival times of the various transmitted frames and the respective signal-to-noise ratios (SNRs), the receiver could correctly receive one of the frames instead of discarding them and losing both [58].

\section{Phy Layer}

IEEE 802.11p makes changes to IEEE 802.11a [59-61]. First of all, the operating frequency is $5.9 \mathrm{GHz}$ instead of $5 \mathrm{GHz}$. Moreover, in the PHY layer of IEEE 802.11a, 5, 10, and $20 \mathrm{MHz}$ bandwidths are available. The 5 and $10 \mathrm{MHz}$ bandwidths can be accomplished by employing a shortened clock/sampling rate. IEEE 802.11a practices the full clocked mode with $20 \mathrm{MHz}$ bandwidth, while IEEE 802.11p employs the half clocked mode with $10 \mathrm{MHz}$ bandwidth. Notwithstanding the bandwidth, the fast Fourier transform (FFT) size is 64, and the amount of subcarriers is 52 (i.e., 48 data subcarriers and four pilot subcarriers). In more detail, Table 2 furnishes a matching of fundamental orthogonal frequency division multiplexing (OFDM) parameters regarding the channel bandwidth.

Table 2. IEEE 802.11 OFDM PHY parameters concerning the channel bandwidth.

\begin{tabular}{|c|c|c|c|}
\hline Paramerers & $\begin{array}{c}20 \mathrm{MHz} \\
\text { Bandwidth }\end{array}$ & $\begin{array}{c}10 \mathrm{MHz} \\
\text { Bandwidth }\end{array}$ & $\begin{array}{c}5 \mathrm{MHz} \\
\text { Bandwidth }\end{array}$ \\
\hline Bit rate $(\mathrm{Mbit} / \mathrm{s})$ & $\begin{array}{c}6,9,12,18 \\
24,36,48,54\end{array}$ & $\begin{array}{c}3,4.5,6,9 \\
12,18,24,27\end{array}$ & $\begin{array}{c}1.5,2.25,3,4.5 \\
6,9,12,13.5\end{array}$ \\
\hline Modulation mode & $\begin{array}{c}\text { BPSK, QPSK, } \\
\text { 16QAM, 64QAM }\end{array}$ & $\begin{array}{c}\text { BPSK, QPSK, } \\
\text { 16QAM, 64QAM }\end{array}$ & $\begin{array}{c}\text { BPSK, QPSK, } \\
\text { 16QAM, 64QAM }\end{array}$ \\
\hline Code rate & $1 / 2,2 / 3,3 / 4$ & $1 / 2,2 / 3,3 / 4$ & $1 / 2,2 / 3,3 / 4$ \\
\hline Number of subcarriers & 52 & 52 & 52 \\
\hline Symbol duration & $4 \mu \mathrm{s}$ & $8 \mu \mathrm{s}$ & $16 \mu \mathrm{s}$ \\
\hline Guard time & $0.8 \mu \mathrm{s}$ & $1.6 \mu \mathrm{s}$ & $3.2 \mu \mathrm{s}$ \\
\hline FFT period & $3.2 \mu \mathrm{s}$ & $6.4 \mu \mathrm{s}$ & $12.8 \mu \mathrm{s}$ \\
\hline Preamble duration & $16 \mu \mathrm{s}$ & $32 \mu \mathrm{s}$ & $64 \mu \mathrm{s}$ \\
\hline Subcarrier spacing & $312.5 \mathrm{kHz}$ & $156.25 \mathrm{kHz}$ & $78.125 \mathrm{kHz}$ \\
\hline
\end{tabular}

As mentioned above, the IEEE 802.11 OFDM PHY defines the IEEE 802.11p OFDM PHY. Nevertheless, there are two critical differences to the transmitter and receiver specifications to sustain the IEEE 802.11p for ITS. They are a significant sterner spectrum mask and more stringent adjacent and non-adjacent channel rejection conditions. These challenging terms are required because the cross channel interference significantly meddles with the transmission when the interferer is nearby to the destination channel and/or when the distance separating the transmitter and receiver is $10 \times$ higher than that between the interferer and receiver. It is valuable to heed that the cross channel interference represents the obstructive impact that transmission in a channel creates on communications in another channel. 
Finally, Table 3 shows the enhanced adjacent and nonadjacent (seldom termed alternate) channel specifications for IEEE 802.11p, and the transmission rates available in a channel; and Table 4 summarizes the differences between IEEE 802.11a and IEEE 802.11p.

Table 3. Transmission rates and channel rejection comparison.

\begin{tabular}{|c|c|c|c|c|c|c|c|c|}
\hline \multirow[b]{2}{*}{ Modulation } & \multirow{2}{*}{$\begin{array}{l}\text { Coding } \\
\text { Rate }\end{array}$} & \multicolumn{2}{|c|}{$\begin{array}{c}\text { Adjacent Channel } \\
\text { Rejection (dB) }\end{array}$} & \multicolumn{2}{|c|}{$\begin{array}{l}\text { Nonadjacent Channel } \\
\text { Rejection (dB) }\end{array}$} & \multirow{2}{*}{$\begin{array}{l}\text { Coded } \\
\text { Bit Rate } \\
\text { (Mbit/s) }\end{array}$} & \multirow{2}{*}{$\begin{array}{c}\text { Data } \\
\text { Rate } \\
\text { (Mbit/s) }\end{array}$} & \multirow{2}{*}{$\begin{array}{c}\text { Data Bits per } \\
\text { OFDM } \\
\text { Symbols }\end{array}$} \\
\hline & & $\begin{array}{c}\text { IEEE } \\
802.11 \\
a / g / n \\
\end{array}$ & $\begin{array}{c}\text { IEEE } \\
\text { 802.11p }\end{array}$ & $\begin{array}{c}\text { IEEE } \\
802.11 \\
a / g / n \\
\end{array}$ & $\begin{array}{c}\text { IEEE } \\
\text { 802.11p }\end{array}$ & & & \\
\hline BPSK & $1 / 2$ & 16 & 28 & 32 & 42 & 6 & 3 & 24 \\
\hline BPSK & $3 / 4$ & 15 & 27 & 31 & 41 & 6 & 4.5 & 36 \\
\hline QPSK & $1 / 2$ & 13 & 25 & 29 & 39 & 12 & 6 & 48 \\
\hline QPSK & $3 / 4$ & 11 & 23 & 27 & 37 & 12 & 9 & 72 \\
\hline 16QAM & $1 / 2$ & 8 & 20 & 24 & 34 & 24 & 12 & 96 \\
\hline 16QAM & $3 / 4$ & 4 & 16 & 20 & 30 & 24 & 18 & 144 \\
\hline 64QAM & $2 / 3$ & 0 & 12 & 16 & 26 & 36 & 24 & 192 \\
\hline 64QAM & $3 / 4$ & -1 & 11 & 15 & 25 & 36 & 27 & 216 \\
\hline
\end{tabular}

Table 4. Differences between IEEE 802.11a and IEEE 802.11p.

\begin{tabular}{ccc}
\hline Parameter & IEEE 802.11a & IEEE 802.11p \\
\hline Sample rate & $20 \mathrm{MHz}$ & $10 \mathrm{MHz}$ \\
\hline Chip duration & $50 \mathrm{~ns}$ & $100 \mathrm{~ns}$ \\
\hline Number of fft points & 64 & 64 \\
\hline Number of subcarriers & $52+\mathrm{DC}$ & $52+\mathrm{DC}$ \\
\hline Number of data subcarriers & 52 & 52 \\
\hline Number of pilot subcarriers & 4 & 4 \\
\hline OFDM symbol period & $T_{\text {Symbols }}=80$ chips $=4 \mu \mathrm{s}$ & $8 \mu \mathrm{s}$ \\
\hline Cyclic prefix & 16 chips $=0.8 \mu \mathrm{s}$ & $1.6 \mu \mathrm{s}$ \\
\hline FFT symbol period & 64 chips $=3.2 \mu \mathrm{s}$ & $1 / 2$ \\
\hline Modulation scheme & BPSK, QPSK, 16QAM, 64QAM & BPSK, QPSK, 16QAM, 64QAM \\
\hline Coding scheme & $1 / 2$ industry convulutional & $3 / 4$ or $2 / 3$ \\
\hline Puncturing & optional puncturing 3/4 or $2 / 3$ & $3,4.5,6,9,12,18,24,27 \mathrm{Mbps}$ \\
\hline Available data rate & $6,9,12,18,24,36,48,54 \mathrm{Mbps}$ & \\
\hline
\end{tabular}

\section{Comparison between IEEE 802.11p and Lte-V2x}

Several papers cited in previous sections facilitate the assessment of a further feature related to the development and dissemination of the IEEE 802.11p protocol concerning vehicular communications; i.e., the comparison with its primary competitor, represented by the LTE-V2V. It is clear that, to date, the IEEE 802.11p is as widespread as the LTE-V2V. There is not a definitive standard for V2V communications, but several car manufacturers have already implemented, in some of their modern cars, the equipment that practices the technology based on the IEEE 802.11p. Nevertheless, it is useful to carry out a comparison between the features related to the IEEE 802.11p and LTE-V2V to understand if one of them is possibly more flexible, more suitable, and better performing in vehicular communications. 
Considering the analysis carried out in ref. [38], the two different technologies are compared in identical external conditions; i.e., in a highway scenario. From the achieved experimental results, it is feasible to acknowledge that the LTE-V2V can deliver a 10\% improvement in the packet reception ratio and a ten times lower update delay compared to the IEEE 802.11p. Anyhow, these results are obtained only in conditions with low data traffic. The situation changes when a more stressful traffic condition, in terms of packets sent and received, is taken into account. In these conditions, while the LTE-V2V performs better concerning the packet reception ratio (ends up 26\% better), the IEEE $802.11 \mathrm{p}$ instead guarantees a lower update delay.

It is beneficial to estimate the results of using different modulation and coding scheme (MCS) strategies that can determine a meaningful impact on both quality of service (QoS) and long-range performance. In ref. [42], it has been determined that the use of IEEE 802.11p allows having outstanding performance up to a few hundred meters, intended as coverage range. It is reasonable to support more than one vehicle every $10 \mathrm{~m}$ with an awareness range of up to 250-300 $\mathrm{m}$. Due to the excessive collision rate, this leads to a decrease in communication reliability in applications with greater distances than those just mentioned. Considering the same conditions, it can be verified that the LTE-V2V standard has reduced performance in short distance communications but is more reliable as the distance increases (for instance, even up to almost $500 \mathrm{~m}$ ) [43].

Finally, it is not conceivable to establish which of the two standards produce the more enhanced performance. Besides, depending on the use conditions and the precise features that the application to be developed requires, it will be feasible to choose one of the standards [61].

\section{IEEE 802.11bd as an Evolution of the IEEE 802.11p}

Before concluding the review carried out in this paper, it is appropriate to perform a brief analysis of the protocol that should be the successor of IEEE 802.11p in the coming years. An IEEE Study Group named Next Generation Vehicular (NGV) was constituted in 2018 to accomplish on an improvement to the IEEE standard for enhanced V2X communication technologies. A task force was appointed to deliver the IEEE 802.11bd, a kind of evolution for IEEE-based V2X communications [62]. This amendment aims at higher spectral efficiency, enhanced reliability, and extended range, while, at the same time, guaranteeing backward adaptability with the existing disposed systems working at $5.9 \mathrm{GHz}$. Thus, in the future, IEEE $802.11 \mathrm{bd}$-based devices could communicate with any IEEE 802.11p-based systems and could be able to transmit in such a way that the IEEE 802.11p devices can receive and decode. Anyhow, it is necessary to note that IEEE 802.11p remains the one deployed. Only when IEEE $802.11 \mathrm{bd}$ is entirely standardized and tested, will future application scenarios be able to seamlessly embrace the next-generation technology.

It will be possible to achieve a smooth transition from "legacy" IEEE 802.11p-based systems to the new standard. IEEE 802.11bd will profit from enduring deployments and infrastructures everywhere in the world by practicing the identical frequency channel without producing an interruption to available ITS stations. In IEEE $802.11 \mathrm{bd}$, the interoperability is accomplished through the employment of a congenial waveform structure. Besides, a well-known channel access mechanism, "listen-before-talk" (i.e., carrier sensing) will be adopted. The asynchronous and non-persistent type of V2X communications applies favorably to conventional V2X networks, where messages are entirely various and non-persistent regarding the size and transmission rate. In fact, the actions triggering the message production are tightly linked to the dynamics of the vehicle [56].

It is profitable to heed that the IEEE 802.11bd task force has proposed several PHY improvements. These involve further rigorous demands on selectivity, sensitivity, and out of band emissions, and the introduction of adaptive repetitions. Other improvements concern the error correction, channel tracking, modulation, data subcarrier, MIMO, range extension, and adaptive retransmissions, to mention a few [63]. All investments delivered in the last years in marshaling IEEE 802.11p will also be ready for IEEE $802.11 \mathrm{bd}$. Additionally, IEEE $802.11 \mathrm{bd}$ advances might be of benefit to the legacy 
IEEE 802.11p and profit from the previously deployed stations. The final version of IEEE 802.11bd should be available on December 2021.

\section{Conclusions}

This paper has presented an overview of the IEEE 802.11p, with distinct attention to ITS, investigating the MAC and PHY layers following the introduction of prefatory notes regarding DSRC. The IEEE 802.11p standard aims to concede a reliable and low-latency communication through the transmission of underlying safety messages among vehicles and roadside infrastructures. This kind of networking could be a crucial step in diminishing road accidents through the exchange of traffic-relevant knowledge in the local environment; for instance, about $500 \mathrm{~m}$ around the vehicle, within a few milliseconds. It is helpful to perceive that the idea of platooning, with networked trucks driving in synchronized convoys, could be based on this technology. Moreover, by way of enhancing road safety and traffic flow, this application on the large scale of the IEEE 802.11p standard could also lessen fuel consumption and carbon dioxide emissions.

The area-wide inception of standardized IEEE 802.11p, which has already been comprehensively examined, could also afford a reliable system for administrators of traffic infrastructure, such as traffic lights, and fleets; i.e., emergency services and construction site vehicles. That way, knowledge of traffic flow, accidents, roadworks, and other relevant circumstances and events can be presumably made accessible in the local area.

Finally, it is essential to record that the CAR 2 CAR Communication Consortium (C2C-CC) and the C-Roads Platform approved a memorandum of understanding for facilitating a narrow agreement among the automotive industry, road governments, and road administrators for planning the deployment of first joining vehicle-to-everything (V2X) services based on IEEE 802.11p. C2C-CC strives on wireless V2V communication applications based on the IEEE 802.11p standard and directs all bids on building standards to guarantee the interoperability of collaborative systems, traversing all vehicle classes across borders and brands. On the contrary, the C-Roads Platform is a shared ambition of the European member states and road directors that are at the stage of the disposing of cooperative intelligent transport systems (C-ITS) for examination and future construction. Preliminary establishments will be arranged over road borders, guaranteeing the interoperability through the collaboration within the C-Roads Platform. Essential factors will be the shared growth of technological specifications that will afford the foundation for all prefatory deployments, and the common cross-site experimentation to deliver interoperability of the arrayed C-ITS services.

Author Contributions: The authors contributed equally to this work. All authors have read and agreed to the published version of the manuscript.

Acknowledgments: This work was related to the D.D. 407 of 27 February 2018 "AIM-Attrazione e Mobilità Internazionale" issued by the Italian Ministry of Education, University, and Research in implementation of Action I.2 "Mobilità dei Ricercatori" Asse I-PON R\&I 2014-2020, taking into account the written amendment procedure of the PON R\&I 2014-2020, pursuant to articles 30 and 90 of Regulation (EU) 1303/2013 started on 21 February 2018 as well as the relevant implementation regulations.

Conflicts of Interest: The authors declare no conflict of interest.

\section{References}

1. Toppan, A.; Bazzi, A.; Toppan, P.; Masini, B.; Andrisano, O. Architecture of a simulation platform for the smart navigation service investigation. In Proceedings of the 2010 IEEE 6th International Conference on Wireless and Mobile Computing, Networking and Communications, Niagara Falls, ON, Canada, 11-13 October 2010; pp. 548-554. [CrossRef]

2. Pau, G.; Severino, A.; Canale, A. Special issue "new perspectives in intelligent transportation systems and mobile communications towards a smart cities context". Future Internet 2019, 11, 228. [CrossRef] 
3. Uchida, N.; Takeuchi, S.; Ishida, T.; Shibata, Y. Mobile traffic accident prevention system based on chronological changes of wireless signals and sensors. J. Wirel. Mob. Netw. Ubiquitous Comput. Dependable Appl. 2017, 8, 57-66. [CrossRef]

4. Bazzi, A.; Zanella, A.; Masini, B. An OFDMA-Based MAC Protocol for Next-Generation VANETs. IEEE Trans. Veh. Technol. 2015, 64, 4088-4100. [CrossRef]

5. Nkenyereye, L.; Tama, B.; Park, Y.; Rhee, K. A fine-grained privacy preserving protocol over attribute based access control for VANETs. J. Wirel. Mob. Netw. Ubiquitous Comput. Dependable Appl. 2015, 6, 98-112.

6. Eziama, E.; Tepe, K.; Balador, A.; Nwizege, K.; Jaimes, L. Malicious Node Detection in Vehicular Ad-Hoc Network Using Machine Learning and Deep Learning. In Proceedings of the 2018 IEEE Globecom Workshops (GC Wkshps), Abu Dhabi, UAE, 9-13 December 2018. [CrossRef]

7. Medeiros, D.; Hernandez, D.; Campista, M.; de Castro P. Pedroza, A. Impact of relative speed on node vicinity dynamics in VANETs. Wirel. Netw. 2019, 25, 1895-1912. [CrossRef]

8. Balador, A.; Uhlemann, E.; Calafate, C.; Cano, J.C. Supporting beacon and event-driven messages in vehicular platoons through token-based strategies. Sensors 2018, 18, 955. [CrossRef]

9. Larue, G.; Watling, C.; Black, A.; Wood, J.; Khakzar, M. Pedestrians distracted by their smartphone: Are in-ground flashing lights catching their attention? A laboratory study. Accid. Anal. Prev. 2020, 134. [CrossRef]

10. Neto, V.; Medeiros, D.; Campista, M. Analysis of mobile user behavior in vehicular social networks. In Proceedings of the 2016 7th International Conference on the Network of the Future (NOF), Buzios, Brazil, 16-18 November 2017. [CrossRef]

11. Pau, G.; Campisi, T.; Canale, A.; Severino, A.; Collotta, M.; Tesoriere, G. Smart pedestrian crossing management at traffic light junctions through a fuzzy-based approach. Future Internet 2018, 10, 15. [CrossRef]

12. Tesoriere, G.; Campisi, T.; Canale, A.; Severino, A. The effects of urban traffic noise on children at kindergarten and primary school: A case study in Enna. AIP Conf. Proc. 2018, 2040, 140005. [CrossRef]

13. Ticali, D.; Denaro, M.; Barracco, A.; Guerrieri, M. Piezoelectric energy harvesting from raised crosswalk devices. AIP Conf. Proc. 2015, 1648, 780006. [CrossRef]

14. Tesoriere, G.; Canale, A.; Severino, A.; Mrak, I.; Campisi, T. The management of pedestrian emergency through dynamic assignment: Some consideration about the "refugee Hellenism" Square of Kalamaria (Greece). AIP Conf. Proc. 2019, 2186, 160004. [CrossRef]

15. Song, F.; Zhu, M.; Zhou, Y.; You, I.; Zhang, H. Smart Collaborative Tracking for Ubiquitous Power IoT in Edge-Cloud Interplay Domain. IEEE Internet Things J. 2019. [CrossRef]

16. Arena, F.; Pau, G. An overview of vehicular communications. Future Internet 2019, 11, 27. [CrossRef]

17. Pei, Q.; Kang, B.; Zhang, L.; Choo, K.R.; Zhang, Y.; Sun, Y. Secure and privacy-preserving 3D vehicle positioning schemes for vehicular ad hoc network. EURASIP J. Wirel. Comm. Netw. 2018, 2018, 271. [CrossRef]

18. Zhang, L.; Luo, M.; Li, J.; Au, M.H.; Choo, K.R.; Chen, T.; Tian, S. Blockchain based secure data sharing system for Internet of vehicles: A position paper. Veh. Commun. 2019, 16, 85-93. [CrossRef]

19. Tesoriere, G.; Campisi, T.; Canale, A.; Severino, A.; Arena, F. Modelling and simulation of passenger flow distribution at terminal of Catania airport. AIP Conf. Proc. 2018, 2040, 140006. [CrossRef]

20. Ticali, D.; Acampa, G.; Denaro, M. Renewable energy efficiency by railway transit. Case study on Rebaudengo railway tunnel in Turin. AIP Conf. Proc. 2018, 2040, 140009. [CrossRef]

21. Arena, F.; Ticali, D. The development of autonomous driving vehicles in tomorrow's smart cities mobility. AIP Conf. Proc. 2018, 2040, 140007. [CrossRef]

22. Giliberto, M.; Arena, F.; Pau, G. A fuzzy-based solution for optimized management of energy consumption in e-bikes. J. Wirel. Mob. Netw. Ubiquitous Comput. Dependable Appl. 2019, 10, 45-64. [CrossRef]

23. Masini, B.; Zuliani, L.; Andrisano, O. On the effectiveness of a GPRS based intelligent transportation system in a realistic scenario. In Proceedings of the 2006 IEEE 63rd Vehicular Technology Conference, Melbourne, Australia, 7-10 May 2006; Volume 6, pp. 2997-3001.

24. Riouali, Y.; Benhlima, L.; Bah, S. Extended Batches Petri Nets Based System for Road Traffic Management in WSNs. J. Sens. Actuator Netw. 2017, 6, 30. [CrossRef]

25. Arena, F.; Pau, G.; Collotta, M. A survey on driverless vehicles: From their diffusion to security features. J. Internet Serv. Inf. Secur. 2018, 8, 1-19. [CrossRef]

26. Ai, Z.; Liu, Y.; Chang, L.; Lin, F.; Song, F. A Smart Collaborative Authentication Framework for Multi-dimensional Fine-grained Control. IEEE Access 2020, 8, 8101-8113. [CrossRef] 
27. Song, F.; Ai, Z.; Zhou, Y.; You, I.; Choo, R.; Zhang, H. Smart Collaborative Automation for Receive Buffer Control in Multipath Industrial Networks. IEEE Trans. Ind. Inform. 2020, 16, 1385-1394. [CrossRef]

28. Song, F.; Zhou, Y.; Wang, Y.; Zhao, T.; You, I.; Zhang, H. Smart Collaborative Distribution for Privacy Enhancement in Moving Target Defense. Inf. Sci. 2019, 479, 593-606. [CrossRef]

29. Song, F.; Zhou, Y.; Chang, L.; Zhang, H. Modeling Space-Terrestrial Integrated Networks with Smart Collaborative Theory. IEEE Netw. 2019, 33, 51-57. [CrossRef]

30. Ai, Z.; Liu, Y.; Song, F.; Zhang, H. A Smart Collaborative Charging Algorithm for Mobile Power Distribution in 5G Networks. IEEE Access 2018, 6, 28668-28679. [CrossRef]

31. Hua, J.; Zhang, R.; Liu, D.; Wang, Y.; Qian, C. Spatial Inequality Analysis of Urban Road Network based on Internet Traffic Data. In Proceedings of the 2018 3rd IEEE International Conference on Intelligent Transportation Engineering (ICITE), Singapore, 3-5 September 2018; pp. 1-5. [CrossRef]

32. Wang, S.; Yuan, J.; Li, X.; Qian, Z.; Arena, F.; You, I. Active Data Replica Recovery for Quality-Assurance Big Data Analysis in IC-IoT. IEEE Access 2019, 7, 106997-107005. [CrossRef]

33. Wang, H.; Wang, L.; Zhou, Z.; Tao, X.; Pau, G.; Arena, F. Blockchain-Based Resource Allocation Model in Fog Computing. Appl. Sci. 2019, 9, 5538. [CrossRef]

34. Balouchestani-Asli, M. Robust Wireless Local Area Networks Based on Compressed Sensing. J. Sens. Actuator Netw. 2018, 7, 15. [CrossRef]

35. Ai, Z.; Zhou, Y.; Song, F. A Smart Collaborative Routing Protocol for Reliable Data Diffusion in IoT Scenarios. Sensors 2018, 18, 1926. [CrossRef]

36. Soares, J.; Silva, N.; Shah, V.; Rodrigues, H. A Road Condition Service Based on a Collaborative Mobile Sensing Approach. In Proceedings of the 2018 IEEE International Conference on Pervasive Computing and Communications Workshops (PerCom Workshops), Athens, Greece, 19-23 March 2018; pp. 639-644. [CrossRef]

37. IEEE. IEEE Standard for Information Technology-Local and Metropolitan Area Networks-Specific Requirements-Part 11: Wireless LAN Medium Access Control (MAC) and Physical Layer (PHY) Specifications Amendment 6: Wireless Access in Vehicular Environments; IEEE Std 802.11p-2010 (Amendment to IEEE Std 802.11-2007 as amended by IEEE Std 802.11k-2008, IEEE Std 802.11r-2008, IEEE Std 802.11y-2008, IEEE Std 802.11n-2009, and IEEE Std 802.11w-2009); IEEE: Piscataway, NJ, USA, 2010; pp. 1-51. [CrossRef]

38. Cecchini, G.; Bazzi, A.; Masini, B.; Zanella, A. Performance comparison between IEEE 802.11p and LTE-V2V in-coverage and out-of-coverage for cooperative awareness. In Proceedings of the 2017 IEEE Vehicular Networking Conference (VNC), Torino, Italy, 27-29 November 2017; pp. 109-114.

39. Harkat, Y.; Amrouche, A.; Lamini, E.S.; Kechadi, M.T. Modeling and performance analysis of the IEEE 802.11p EDCA mechanism for VANET under saturation traffic conditions and error-prone channel. AEU Int. J. Electron. Commun. 2019, 101, 33-43. [CrossRef]

40. Cao, S.; Lee, V.C. An accurate and complete performance modeling of the IEEE 802.11p MAC sublayer for VANET. Comput. Commun. 2020, 149, 107-120. [CrossRef]

41. Ramanathan, R. An Empirical study on MAC layer in IEEE 802.11p/WAVE based Vehicular Ad hoc Networks. Procedia Comput. Sci. 2018, 143, 720-727. [CrossRef]

42. Chen, S.; Nai, W.; Dong, D.; Zheng, W.; Jing, W. Key Indices Analysis of IEEE 802.11p based Vehicle to Infrastructure System in Highway Environment. Procedia Soc. Behav. Sci. 2013, 96, 188-195. [CrossRef]

43. Bazzi, A.; Masini, B.M.; Zanella, A.; Thibault, I. On the Performance of IEEE 802.11p and LTE-V2V for the Cooperative Awareness of Connected Vehicles. IEEE Trans. Veh. Technol. 2017, 66, 10419-10432. [CrossRef]

44. Bazzi, A.; Zanella, A.; Masini, B. A distributed virtual traffic light algorithm exploiting short range V2V communications. Ad Hoc Netw. 2016, 49, 42-57. [CrossRef]

45. Arena, F.; Pau, G.; Severino, A. V2X Communications Applied to Safety of Pedestrians and Vehicles. J. Sens. Actuator Netw. 2019, 9, 3. [CrossRef]

46. Silva, C.; Silva, L.; Santos, L.; Sarubbi, J.; Pitsillides, A. Broadening understanding on managing the communication infrastructure in vehicular networks: Customizing the coverage using the Delta Network. Future Internet 2018, 11, 1. [CrossRef]

47. Sarubbi, J.; Silva, T.; Martins, F.; Wanner, E.; Silva, C. Allocating Roadside Units in VANETs Using a Variable Neighborhood Search Strategy. In Proceedings of the 2017 IEEE 85th Vehicular Technology Conference (VTC Spring), Sydney, Australia, 4-7 June 2017. [CrossRef] 
48. Abboud, K.; Omar, H.A.; Zhuang, W. Interworking of DSRC and Cellular Network Technologies for V2X Communications: A Survey. IEEE Trans. Veh. Technol. 2016, 65, 9457-9470. [CrossRef]

49. Fitah, A.; Badri, A.; Moughit, M.; Sahel, A. Performance of DSRC and WIFI for Intelligent Transport Systems in VANET. Procedia Comput. Sci. 2018, 127, 360-368. [CrossRef]

50. Zhou, P.; Liu, Y.; Wang, J.; Deng, W.; Oh, H. Performance analysis of prioritized broadcast service in WAVE/IEEE 802.11p. Comput. Netw. 2016, 107, 233-245. [CrossRef]

51. Shahen Shah, A.F.M.; Ilhan, H.; Tureli, U. Modeling and Performance Analysis of the IEEE 802.11P MAC for VANETs. In Proceedings of the 2019 42nd International Conference on Telecommunications and Signal Processing (TSP), Budapest, Hungary, 1-3 July 2019; pp. 393-396. [CrossRef]

52. Song, H.; Lee, H.S. An overview of analytic models of the IEEE 802.11p MAC protocol for vehicle-to-vehicle safety communications and their technical issues. In Proceedings of the 2013 International Conference on ICT Convergence (ICTC), Jeju Island, Korea, 14-16 October 2013; pp. 612-613. [CrossRef]

53. Yao, Y.; Hu, Y.; Yang, G.; Zhou, X. On MAC Access Delay Distribution for IEEE 802.11p Broadcast in Vehicular Networks. IEEE Access 2019, 7, 149052-149067. [CrossRef]

54. Wang, L.; Iida, R.F.; Wyglinski, A.M. Performance Analysis of EDCA for IEEE 802.11p/DSRC Based V2V Communication in Discrete Event System. In Proceedings of the 2017 IEEE 86th Vehicular Technology Conference (VTC-Fall), Toronto, ON, Canada, 24-27 September 2017; pp. 1-5. [CrossRef]

55. Bazzi, A.; Cecchini, G.; Menarini, M.; Masini, B.M.; Zanella, A. Survey and Perspectives of Vehicular Wi-Fi versus Sidelink Cellular-V2X in the 5G Era. Future Internet 2019, 11, 122. [CrossRef]

56. Naik, G.; Choudhury, B.; Park, J. IEEE 802.11bd 5G NR V2X: Evolution of Radio Access Technologies for V2X Communications. IEEE Access 2019, 7, 70169-70184. [CrossRef]

57. Sjoberg, K.; Uhlemann, E.; Strom, E.G. How Severe Is the Hidden Terminal Problem in VANETs When Using CSMA and STDMA? In Proceedings of the 2011 IEEE Vehicular Technology Conference (VTC Fall), San Francisco, CA, USA, 15-18 September 2011; pp. 1-5. [CrossRef]

58. Wijngaarden, P.; Eenennaam, M. Frame Capture in IEEE 802.11p Vehicular Networks. In Proceedings of the Second Workshop on the Pervasive Application of Wireless Technologies, Twente, Netherlands, September 2010.

59. Anwar, W.; Kulkarni, K.; Augustin, T.R.; Franchi, N.; Fettweis, G. PHY Abstraction Techniques for IEEE 802.11p and LTE-V2V: Applications and Analysis. In Proceedings of the 2018 IEEE Globeco Workshops (GC Wkshps), Abu Dhabi, UAE, 9-13 December 2018; pp. 1-7. [CrossRef]

60. Saponara, S.; Gagliardi, A. Modeling and Analysis of 802.11p Physical Layer for V2X Connected Transport Systems Considering Harsh Operating Conditions and HW Device Performance. In Proceedings of the 2018 International Conference of Electrical and Electronic Technologies for Automotive, Milano, Italy, 9-11 July 2018; pp. 1-6. [CrossRef]

61. Moller, A.; Nuckelt, J.; Rose, D.M.; Kurner, T. Physical Layer Performance Comparison of LTE and IEEE 802.11p for Vehicular Communication in an Urban NLOS Scenario. In Proceedings of the 2014 IEEE 80th Vehicular Technology Conference (VTC2014-Fall), Vancouver, BC, Canada, 14-17 September 2014; pp. 1-5. [CrossRef]

62. Status of Project IEEE P802.11bd. Available online: http://www.ieee802.org/11/Reports/tgbd_update.htm (accessed on 18 April 2020).

63. Anwar, W.; Franchi, N.; Fettweis, G. Physical Layer Evaluation of V2X Communications Technologies: 5G NR-V2X, LTE-V2X, IEEE 802.11bd, and IEEE 802.11p. In Proceedings of the 2019 IEEE 90th Vehicular Technology Conference (VTC2019-Fall), Honolulu, HI, USA, 21-25 September 2019; pp. 1-7.

(C) 2020 by the authors. Licensee MDPI, Basel, Switzerland. This article is an open access article distributed under the terms and conditions of the Creative Commons Attribution (CC BY) license (http:// creativecommons.org/licenses/by/4.0/). 